\title{
Improving Spatial Detection of Twins Achieved by Measuring Individual Kikuchi Band Intensity in EBSD Patterns
}

\author{
Travis Rampton ${ }^{1}$, David Fullwood ${ }^{2}$, Stuart Wright ${ }^{3}$. \\ 1. EDAX, Mahwah, NJ, USA \\ 2. Department of Mechanical Engineering, Brigham Young University, Provo, UT, USA \\ 3. EDAX, Draper, UT, USA
}

Twin boundaries in a crystalline material can be defined by a particular rotation angle about a particular access or a mirrored crystal orientation about a particular plane [1]. For example, copper twins are typically defined by a $60^{\circ}$ rotation about $\left.<111\right\rangle$ where the associated twin plane is of the $\{111\}$ family. One critical area of twin research looks at deformation twinning as the limiting factor for formability of Mg alloys, such as AZ31 [2]. In AZ31 there are two basic twin modes: compression twinning and tension twinning. The latter phenomenon forms fairly large, easy to detect twinned regions within parent grains, whereas the former tends to form extremely thin twins that are on the order of $100 \mathrm{~nm}$ wide. Additionally, the copper which is frequently seen in many microelectronics contains twins on the order of $10 \mathrm{~nm}$ [3]. In both cases these features are within the detectable limits for a modern scanning electron microscope (SEM). However, identifying these twins via crystal orientation relations with electron backscatter diffraction (EBSD) in the SEM relies on a larger spatial resolution which makes detecting these twins from crystallographic information difficult in the SEM. This study presents a method whereby improved spatial resolution of thin twins can be achieved with EBSD.

EBSD data is produced when the electron beam of an SEM diffracts off the surface of a crystalline material and forms a pattern of Kikuchi bands on the EBSD detector. The captured EBSD pattern is assigned an orientation, or indexed, based on the pattern of Kikuchi bands which correspond to crystal planes. This diffraction information emanates from the interaction volume of the electron beam which is inversely proportional to the density of the material and thus less dense materials typically end up having lower spatial resolution. The physical interaction volume size can result in a weak EBSD pattern containing data from both the twin and its surrounding parent crystal. When diffraction signals mix in EBSD the result in low image quality (IQ) which is a measure of diffraction strength [4]. Drops in IQ are quite typical near grain boundaries, twin boundaries, and surface defects. For this study the data extraction will proceed in the following manner:

- Areas of low IQ will be used to identify potential sites of twin boundaries in a microstructure.

- Individual Kikuchi band intensities will be measured from EBSD patterns in the regions of interest.

- Kikuchi band intensities will be analyzed for consistent intensity.

- Bands of consistent intensity will be correlated to crystal planes to check if they match on of the crystal planes related to twinning.

Following these steps proof of concept will also be demonstrated on Inconel 600 and Ta with large, easily detectable twins. Figure 1 shows a set of Kikuchi band intensity profiles taken across an Inconel 600 sample. This study will then identify thin twins in $\mathrm{Mg}$ and $\mathrm{Cu}$ where traditional EBSD could not due to spatial limitations. Figure 2 shows an example area of a twin only detected after applying the diffraction band intensity method. 


\section{References}

[1] J. W. Christian et al, Progress in materials science, 39 (1995), pp. 1-157.

[2] J. Scott et al, Met Trans A, 2013.

[3] D. Chen et al, J Microsc, 236 (2009), pp. 44-51.

[4] S. I. Wright et al, Microscopy and Microanalysis, 12 (2006), pp. 72-84.
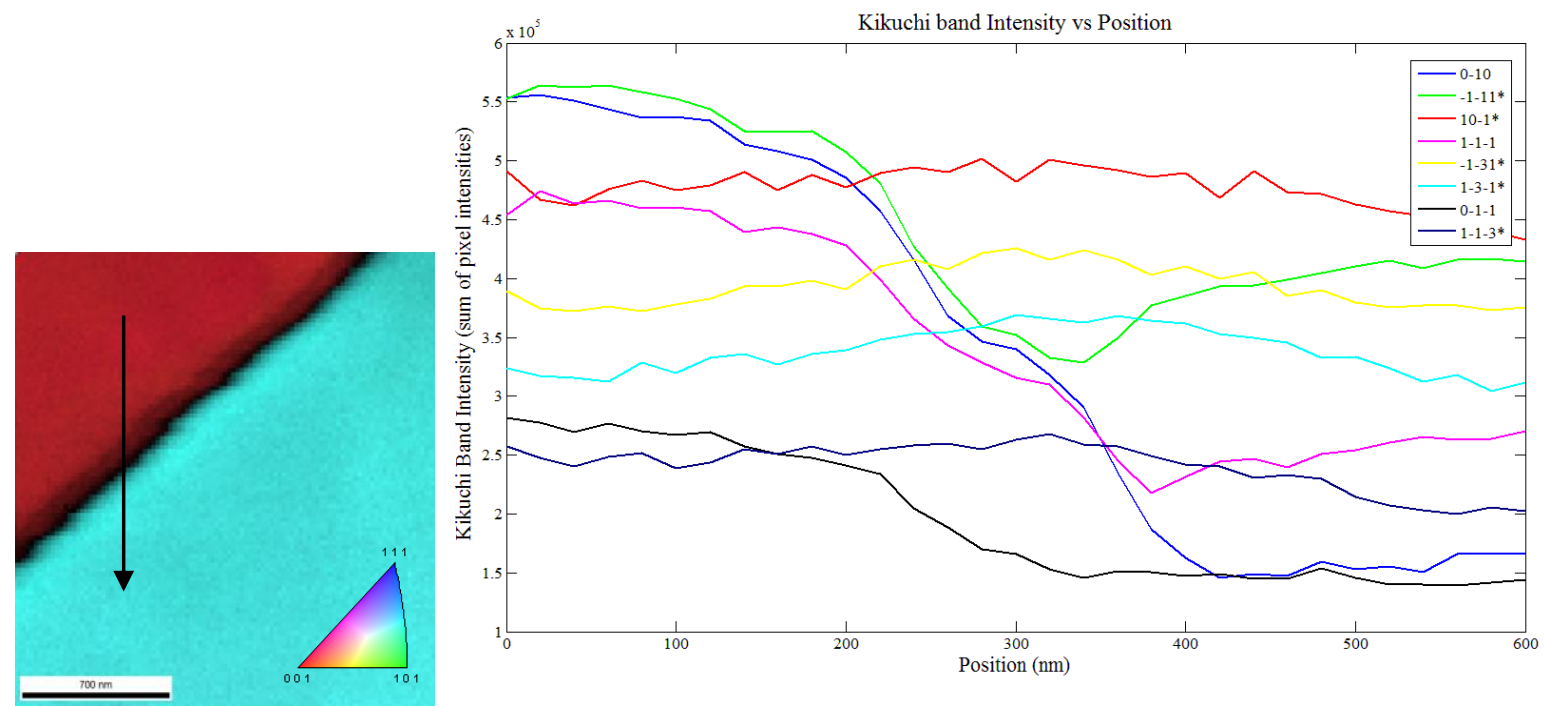

Figure 1. (Left) Inverse pole figure and (right) IQ and vertical Kikuchi band intensity across twin boundary. Crystal planes associated with each Kikuchi band are labeled.

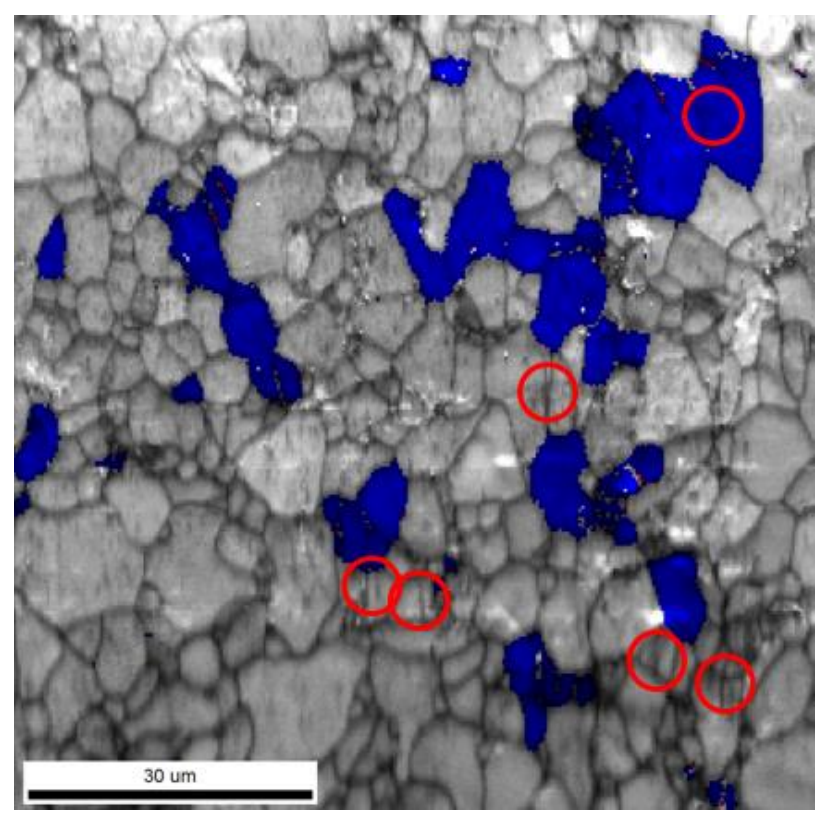

Figure 2. IQ map and twin/parent map. Parent grains detected by standard EBSD are blue and the associated twins are red. A few twins detected by the new band intensity method are circled. 